Brief paper

\title{
Equivalence between classes of multipliers for slope-restricted nonlinearities
}

\author{
Joaquin Carrasco ${ }^{1}$, William P. Heath, Alexander Lanzon \\ Control Systems Centre, School of Electrical and Electronic Engineering, The University of Manchester, Sackville Street Building, Manchester M13 9PL, UK
}

\section{A R T I C L E I N F O}

\section{Article history:}

Received 31 March 2012

Received in revised form

21 September 2012

Accepted 9 January 2013

Available online 13 March 2013

\section{Keywords:}

Slope-restricted nonlinearities

Zames-Falb multipliers

Absolute stability

\begin{abstract}
A B S T R A C T
Different classes of multipliers have been proposed in the literature for obtaining stability criteria using passivity theory, integral quadratic constraint (IQC) theory or Lyapunov theory. Some of these classes of multipliers can be applied with slope-restricted nonlinearities. In this paper the concept of phasecontainment is defined and it is shown that several classes are phase-contained within the class of Zames-Falb multipliers. There are two main consequences: firstly it follows that the class of Zames-Falb multipliers remains, to date, the widest class of available multipliers for slope-restricted nonlinearities; secondly further restrictions may be avoided when exploiting the parametrization of the other classes of multipliers.
\end{abstract}

(C) 2013 Elsevier Ltd. All rights reserved.

\section{Introduction}

The investigation of absolute stability for the system in Fig. 1, where $G$ is a linear time-invariant (LTI) system and $\phi$ is a nonlinearity within a given class, is known as the Lur'e problem. For example, if one would like to investigate the stability of a feedback control system with saturation in the actuator, the closed-loop could be expressed as Fig. 1. Because the saturation belongs to the class of so-called sector bounded nonlinearities, simple analysis conditions based upon the LTI part of the system can be derived, i.e. strictly positive realness (Khalil, 2002), and applied to anti-windup synthesis (Mulder, Kothare, \& Morari, 2001). However the conditions are inherently conservative. In order to reduce such conservatism, saturation can be more efficiently described as a slope-restricted and odd nonlinearity; multiplier techniques may then be used.

In Desoer and Vidyasagar (1975) and Zames and Falb (1968), the classical multiplier approach is developed for any general application (see Fig. 2). Loosely speaking, if $M$ has a canonical factorization, then positivity of $M G$ and $M^{*} \phi$ is enough to prove stability.

\footnotetext{
This work has been founded by EPSRC Grant EP/H01600/1. The material in this paper was presented at the 51st IEEE Conference on Decision and Control (CDC), December 10-13, 2012, Maui, Hawaii, USA. This paper was recommended for publication in revised form by Associate Editor Nathan Van De Wouw under the direction of Editor Andrew R. Teel.

E-mail addresses: joaquin.carrascogomez@manchester.ac.uk (J. Carrasco), William.Heath@manchester.ac.uk (W.P. Heath), a.lanzon@ieee.org (A. Lanzon).

1 Tel.: +440161306 2290; fax: +440161306 4648 .
}

The multiplier technique can be used for applying either passivity theory (Zames \& Falb, 1968) or integral quadratic constraint (IQC) theory (Megretski \& Rantzer, 1997). Although the multiplier is not directly used when Lyapunov theory is used, the results can also be interpreted in terms of multipliers (Brockett \& Willems, 1965) by using the path integral approach (see Gruber, 1969; Willems, 1998). In summary, given a class of nonlinearities, a class of multipliers preserving the positivity of the class of nonlinearities must be defined; then for a particular linear system, stability of the feedback interconnection of Fig. 1 is ensured if an element of the class of multipliers can be found such that $M G$ is positive.

In this paper, we are concerned with nonlinearities that are slope-restricted. In particular, for SISO slope-restricted and odd nonlinearities several classes of multipliers were proposed in the Sixties, summarized by Barabanov (1988). The celebrated paper (Zames \& Falb, 1968) appears to give the best description of the class of multipliers, including RL and RC multipliers (Falb \& Zames, 1968). However more conservative graphical criteria, such as the circle (Zames, 1966), Popov (Popov, 1961) or off-axis circle criteria (Cho \& Narendra, 1968), were developed due to the lack of tools for searching for Zames-Falb multipliers.

As appropriate computation tools have become available, several works (Carrasco, Heath, Li, \& Lanzon, 2012; Chang, Mancera, \& Safonov, 2011; Chen \& Wen, 1995; Gapski \& Geromel, 1994; Safonov \& Wyetzner, 1987; Turner, Kerr, \& Postlethwaite, 2009) have proposed different searches within the class of Zames-Falb multipliers. Meanwhile several authors propose extensions to the Zames-Falb class. Jönsson (1997) extends the class of Zames-Falb multipliers by adding a Popov term, and Altshuller (2011) extends 


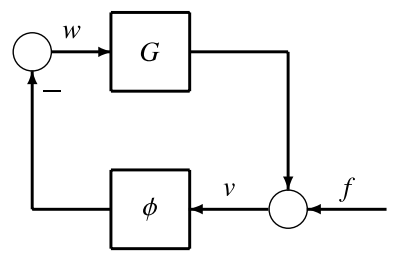

Fig. 1. Lur'e problem. Stability conditions are sought in terms of the characterization of the nonlinearity $\phi$ and the frequency properties of the LTI system $G$.

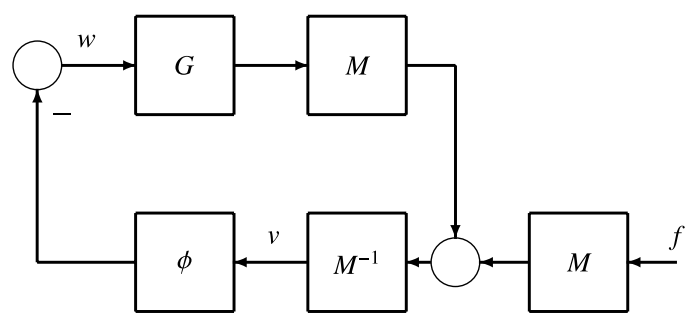

Fig. 2. Multiplier transformation. The class of multipliers is designed to preserve the positivity of $\phi$.

the class of Zames-Falb multipliers by adding a quadratic term. Park (2002) proposes an LMI search for certain MIMO multipliers and discusses their relation to the Zames-Falb multipliers in the SISO case; the SISO version of Park (2002) is also given by Barabanov (1988), and was introduced by Yakubovich (1965) via a Lyapunov approach and by Dewey and Jury (1966) via an input-output approach.

Dynamic multipliers such as Zames-Falb multipliers have been used as an analysis tool (D'Amato, Rotea, Megretski, \& Jönsson, 2001; Heath \& Wills, 2007; Kothare \& Morari, 1999). Although their use in synthesis is proposed by, for example, Kerr, Turner, Villota, Jayasuriya, and Postlethwaite (2011); Moreno, Baños, and Berenguel (2010); and Veenman and Scherer (2011), it is not yet understood how to exploit their full generality; more conservative criteria are still in use for antiwindup synthesis.

In Kulkarni and Safonov (2002a), the class of Zames-Falb multipliers is stated to be the most general class of biproper multipliers. However some classes of multipliers are improper, e.g. Popov multipliers. The aim of this paper is to demonstrate that the Zames-Falb class is the widest available class of multipliers for slope-restricted nonlinearities. Firstly, we define notions of phasecontainment and phase-equivalence. Secondly, we show that Popov multipliers, Park's multipliers (Park, 2002), and the extensions of the Zames-Falb multipliers are all phase-contained within the class of Zames-Falb multipliers. Our treatment is consistent with that of Falb and Zames (1968) who showed a similar relation for RC and RL multipliers. Note that while the Popov and RL and RC classes of multipliers have been described as belonging to (limiting) subsets of the class of Zames-Falb multipliers (e.g. Kulkarni \& Safonov, 2002 b), such statements have not been rigorously proven; nor are they necessarily correct in all cases.

The most important consequences of this paper are:

- To date, Zames-Falb multipliers are the widest class for analysing the stability of the class of slope-restricted nonlinearities. If a new class of multipliers is proposed containing multipliers which are not Zames-Falb multipliers, it should not necessarily be concluded that this new class is wider.

- Stability results for slope-restricted nonlinearities using the multipliers under discussion can be stated as corollaries of the Zames-Falb theorem. Therefore no extra conditions are needed and $\mathscr{L}_{2}$ stability is obtained.
- Since the passivity theory and IQC theory are equivalent for Zames-Falb multipliers (Carrasco, Heath, \& Lanzon, 2012), it follows that the theories are also equivalent for slope-restricted nonlinearities using any of the multipliers under discussion.

The structure of the paper is as follows. After some preliminary results in Section 2, we define our notions of phase-containment and phase-equivalence in Section 3. These definitions are used in Sections 4-6 for demonstrating different equivalences between classes of multipliers. The usefulness of the classes of multipliers is discussed in Section 7 and finally, conclusions are stated in Section 8.

\section{Notation and preliminary results}

Let $\mathcal{L}_{2}^{m}[0, \infty)$ be the Hilbert space of all square integrable and Lebesgue measurable functions $f:[0, \infty) \rightarrow \mathbb{R}^{m}$. Similarly, $\mathcal{L}_{2}^{m}$ $(-\infty, \infty)$ can be defined for $f:(-\infty, \infty) \rightarrow \mathbb{R}^{m}$. A truncation of the function $f$ at $T$ is given by $f_{T}(t)=f(t) \forall t \leq T$ and $f_{T}(t)=$ $0 \forall t>T$. The function $f$ belongs to the extended space $\mathcal{L}_{2 e}^{m}[0, \infty)$ if $f_{T} \in \mathcal{L}_{2}^{m}[0, \infty)$ for all $T>0$. In addition, $\mathcal{L}_{1}(-\infty, \infty)$ (henceforth $\mathscr{L}_{1}$ ) is the space of all absolute integrable functions; given a function $h: \mathbb{R} \rightarrow \mathbb{R}$ such that $h \in \mathcal{L}_{1}$, its $\mathcal{L}_{1}$-norm is given by

$\|h\|_{1}=\int_{-\infty}^{\infty}|h(t)| d t$

A nonlinearity $\phi: \mathscr{L}_{2 e}[0, \infty) \rightarrow \mathscr{L}_{2 e}[0, \infty)$ is said to be memoryless if there exists $N: \mathbb{R} \rightarrow \mathbb{R}$ such $(\phi v)(t)=N(v(t))$ for all $t \in \mathbb{R}$. Henceforth we assume that $N(0)=0$. A memoryless nonlinearity $\phi$ is said to be bounded if there exists a positive constant $C$ such that $|N(x)|<C|x|$ for all $x \in \mathbb{R}$. The nonlinearity $\phi$ is said to be monotone if for any two real numbers $x_{1}$ and $x_{2}$ we have

$0 \leq \frac{N\left(x_{1}\right)-N\left(x_{2}\right)}{x_{1}-x_{2}}$.

Moreover, $\phi$ is said to be slope-restricted or incrementally bounded in the sector $S[0, k]$, (henceforward we write $\phi_{k}$ ), if

$0 \leq \frac{N\left(x_{1}\right)-N\left(x_{2}\right)}{x_{1}-x_{2}} \leq k$

for all $x_{1} \neq x_{2}$. The nonlinearity $\phi$ is said to be odd if $N(x)=$ $-N(-x)$ for all $x \in \mathbb{R}$.

This paper focuses the stability of the feedback interconnection of a stable LTI system $G$ and a slope-restricted nonlinearity $\phi_{k}$, represented in Fig. 1 and given by

$\left\{\begin{array}{l}v=f+G w \\ w=-\phi_{k} v\end{array}\right.$

Since $G$ is a stable LTI system, the exogenous input in this part of the loop can be taken as the zero signal without loss of generality. It is well-posed if the map $(v, w) \mapsto(0, f)$ has a causal inverse on $\mathcal{L}_{2 e}^{2}[0, \infty)$; this interconnection is $\mathcal{L}_{2}$-stable if for any $f \in \mathscr{L}_{2}[0, \infty)$, then $G w \in \mathcal{L}_{2}[0, \infty)$ and $\phi_{k} v \in \mathcal{L}_{2}[0, \infty)$, and it is absolutely $\mathscr{L}_{2}$-stable if it is $\mathscr{L}_{2}$-stable for all $\phi_{k}$ within the class of nonlinearities. In addition, $G(j \omega)$ means the transfer function of the LTI system $G$. Finally, given an operator $M$, then $M^{*}$ means its $\mathcal{L}_{2}$-adjoint (see Desoer \& Vidyasagar, 1975 for a definition). For LTI systems, $M^{*}(s)=M^{\top}(-s)$, where ${ }^{\top}$ means transpose.

The standard notation $\mathbf{L}_{\infty}\left(\mathbf{R L}_{\infty}\right)$ is used for the space of all (proper real rational) transfer functions bounded on the imaginary axis and infinity; $\mathbf{R H}_{\infty}$ is used for the space of all proper real rational transfer functions such that all their poles have strictly negative real parts; and $\mathbf{R} \mathbf{H}_{\infty}^{\perp}$ is used for the space of all proper real rational transfer functions such that all their poles have strictly 
positive real parts. The $H_{\infty}$-norm of a SISO transfer function $G$ is defined as

$\|G\|_{\infty}=\sup _{\omega \in \mathbb{R}}(|G(j \omega)|)$.

With some acceptable abuse of notation, given a rational strictly proper transfer function $H(s)$ bounded on the imaginary axis, $\|H\|_{1}$ means the $\mathcal{L}_{1}$-norm of the impulse response of $H(s)$.

\subsection{Zames-Falb theorem}

The following theorem provides the $\mathscr{L}_{2}$-stability of system (4) subject to the existence of an appropriate Zames-Falb multiplier.

Theorem 2.1 (Zames E Falb, 1968). Consider the feedback system in Fig. 1 with $G \in \mathbf{R H}_{\infty}$, and a nonlinearity $\phi_{k-\epsilon}$ slope-restricted in $S[0, k-\epsilon]$ for some $\epsilon>0$. Assume that the feedback interconnection is well-posed. Then suppose that there exists a convolution operator $M: \mathscr{L}_{2}(-\infty, \infty) \rightarrow \mathscr{L}_{2}(-\infty, \infty)$ whose impulse response is of the form

$m(t)=\delta(t)-\sum_{i=1}^{\infty} z_{i} \delta\left(t-t_{i}\right)-z_{a}(t)$,

where $\delta$ is the Dirac delta function and

$\sum_{i=0}^{\infty}\left|z_{i}\right|<\infty, \quad z_{a} \in \mathcal{L}_{1}$, and $t_{i} \in \mathbb{R} \forall i \in \mathbb{N}$.

Assume that:

(i)

$$
\left\|z_{a}\right\|_{1}+\sum_{i=0}^{\infty}\left|z_{i}\right|<1
$$

(ii) either $z_{a}(t) \geq 0$ for all $t \in \mathbb{R}$ and $z_{i} \geq 0$ for all $i \in \mathbb{N}$, or $\phi_{k-\epsilon}$ is odd; and

(iii) there exists $\delta>0$ such that

$\operatorname{Re}\{M(j \omega)(1+k G(j \omega))\} \geq \delta \quad \forall \omega \in \mathbb{R}$.

Then the feedback interconnection (4) is $\mathcal{L}_{2}$-stable.

\subsection{Zames-Falb multipliers}

Eqs. (6)-(8) in Theorem 2.1 provide the class of Zames-Falb multipliers. It is a subset of $\mathbf{L}_{\infty}$, i.e. it is not limited to rational transfer functions. However in many parts of this paper we restrict our attention to such rational multipliers, i.e. we set $z_{i}=0$ for all $i \in \mathbb{N}$.

Definition 2.2. The class of SISO rational Zames-Falb multipliers $\mathcal{M}$ contains all SISO rational transfer functions $M \in \mathbf{R L}_{\infty}$ such that $M(s)=1-Z(s)$, where $Z(s)$ is a rational strictly proper transfer function and $\|Z\|_{1}<1$.

Lemma 2.3 (Carrasco, Heath, Li et al., 2012). Let $M \in \mathbf{R L}_{\infty}$ be a rational transfer function with $M(s)=M(\infty)+\widehat{M}(s)$, where $\widehat{M}(s)$ denotes its associated strictly proper transfer function. Then $M(s)$ is a Zames-Falb multiplier if and only if $\|\widehat{M}\|_{1}<M(\infty)$.

Remark 2.4. The corresponding lemma given in Carrasco, Heath, Li et al.(2012) is limited to $M \in \mathbf{R H}_{\infty}$, but its extension to $M \in \mathbf{R L}_{\infty}$ is straightforward.

In this paper, we will often consider first order Zames-Falb multipliers, i.e. multipliers represented by a first order transfer function. These are by implication rational. It is worth noting that the impulse response of a first order Zames-Falb multiplier is always positive. Hence in this case condition (ii) in Theorem 2.1 is always satisfied and the odd condition on $\phi_{k-\epsilon}$ is not needed.
A multiplier $M \in \mathbf{R H}_{\infty}$, with impulse response supported on the positive time axis is termed a causal multiplier. A multiplier $M \in \mathbf{R H}_{\infty}^{\perp}$ with impulse response supported on the negative time axis is called an anticausal multiplier. Otherwise, the multiplier is called noncausal.

\subsection{Practicalities and absolute stability}

In the original theorem of Zames and Falb (1968), when the linear condition (9) holds for some constant $k$, the nonlinearity is required to belong to the sector $S[0, k-\epsilon]$, where $\epsilon$ can be arbitrarily small but strictly positive. Nevertheless, its extension to the sector $S[0, k]$ is trivial since $\|M\|_{\infty}<1$ (see Carrasco, Heath, Li et al., 2012; Kulkarni \& Safonov, 2002a); thus Theorem 2.1 actually holds under the weaker assumption that $\epsilon \leq 0$. A prior lemma is needed.

Lemma 2.5. Let $M \in \mathbf{L}_{\infty}$ be a Zames-Falb multiplier satisfying equations (6)-(8). Assume that (9) is satisfied for $G \in \mathbf{R H}_{\infty}, M, k>0$, and $\delta>0$. Then there exist $\xi>0$ and $\delta_{1}>0$ such that

$\operatorname{Re}\{M(j \omega)(1+(k+\xi) G(j \omega))\} \geq \delta_{1}>0 \quad \forall \omega \in \mathbb{R}$.

Proof. Choose $\xi=\frac{\delta}{2\|G\|_{\infty}}$. Then

$$
\begin{aligned}
& \operatorname{Re}\left\{M(j \omega)\left(1+\left(k+\frac{\delta}{2\|G\|_{\infty}}\right) G(j \omega)\right)\right\} \\
& =\operatorname{Re}\{M(j \omega)(1+k G(j \omega))\}+\frac{\delta}{2\|G\|_{\infty}} \operatorname{Re}\{M(j \omega) G(j \omega)\}
\end{aligned}
$$

Taking into account that $\|M\|_{\infty}<1$ and (9), it follows that

$\operatorname{Re}\{M(j \omega)(1+(k+\xi) G(j \omega))\} \geq \delta_{1}>0 \quad \forall \omega \in \mathbb{R}$,

where $\delta_{1}=\frac{\delta}{2}$.

The significance of Theorem 2.1 is that it can be applied when the nonlinearity is characterized to be memoryless, sloperestricted, and odd (in some cases). An absolute stability result can be stated as follows:

Corollary 2.6. Consider the feedback system in Fig. 1 with $G \in \mathbf{R H}_{\infty}$ and any nonlinearity $\phi_{k}$ slope-restricted in $S[0, k]$. Assume that the system is well-posed. Then suppose that there exists $M \in \mathcal{M}$ such that:

(i) either $\phi_{k}$ is odd or the inverse Laplace transform of $H(s)=M(s)$ -1 is negative for all $t \in \mathbb{R}$; and

(ii) there exists $\delta>0$ such that

$$
\operatorname{Re}\{M(j \omega)(1+k G(j \omega))\} \geq \delta \quad \forall \omega \in \mathbb{R} .
$$

Then the feedback interconnection (4) is absolutely stable.

In this paper, we compare different criteria for absolute stability. If a criterion guarantees the stability of feedback of $G \in$ $\mathbf{R H}_{\infty}$ and any nonlinearity $\phi_{k}$ slope-restricted in $S[0, k]$, then the linear feedback interconnection of $G(s)$ and any linear gain $0 \leq$ $K \leq k$ must be stable. The following definition is used in works that focus on stability criteria, e.g. Chen and Wen (1995); and Safonov and Wyetzner (1987).

Definition 2.7. Given $G \in \mathbf{R H}_{\infty}$, the Nyquist value $k_{N}$ is the supremum of the values $k$ such that $K G(s)$ satisfies the Nyquist Criterion for all $K \in[0, k]$, i.e.

$k_{N}=\sup \left\{k \in \mathbb{R}^{+}: \inf _{\omega}\{|1+K G(j \omega)|\}>0 \forall K \in[0, k]\right\}$.

As a result, we can restrict our attention to a subset of $\mathbf{R} \mathbf{H}_{\infty}$ without loss of generality. This will be essential to prove the relationship between Zames-Falb multipliers and Popov multipliers. 
Definition 2.8. The subset $\varsigma \mathcal{R} \subset \mathbf{R H}_{\infty}$ is defined as follows

$§ \mathcal{R}=\left\{\widehat{G} \in \mathbf{R H}_{\infty}: \widehat{G}^{-1} \in \mathbf{R H}_{\infty}\right.$ and $\left.\widehat{G}(\infty)>0\right\}$.

Lemma 2.9. Assume that the feedback interconnection in Fig. 1 with $G \in \mathbf{R H}_{\infty}$ and any nonlinearity $\phi_{k}$ slope-restricted $S[0, k]$ is $\mathscr{L}_{2}$ stable. Then $(1+k G) \in \& \mathcal{R}$.

It follows that if $\widehat{G}=1+k G \notin 8 \mathcal{R}$, with $G \in \mathbf{R} \mathbf{H}_{\infty}$ and $k>0$, then the feedback interconnection of $G$ and the class of nonlinearities slope-restricted in $S[0, k]$ cannot be absolutely $\mathcal{L}_{2}$ stable.

\section{Equivalence of multipliers}

In the literature of SISO slope-restricted nonlinearities, several classes of multipliers have been defined. The equivalence between specific classes is discussed by Falb and Zames (1968); Kulkarni and Safonov (2002b) but in neither is a general concept of equivalence rigorously defined. Similarly, alternative definitions of the Popov multiplier in the early literature (Brockett \& Willems, 1965) implicitly assume such equivalence. In the following, we define the terms phase-substitute, phase-contained, and phase-equivalent with respect to classes of multipliers.

Our notion of phase-containment for a class of nonlinearities is made with respect to an appropriate set of LTI systems. We restrict our attention to the set $₫ \mathcal{R}$, which by Lemma 2.9 is naturally associated with slope-restricted nonlinearities.

For slope-restricted nonlinearities we can define the concept of phase-substitution as follows:

Definition 3.1. Let $M_{a}$ and $M_{b}$ be two multipliers and $\widehat{G} \in \& \mathcal{R}$. The multiplier $M$ is a phase-substitute of the multiplier $M_{a}$ when

$\operatorname{Re}\left\{M_{a}(j \omega) \widehat{G}(j \omega)\right\} \geq \delta_{1} \quad \forall \omega \in \mathbb{R}$

for some $\delta_{1}>0$ implies

$\operatorname{Re}\left\{M_{b}(j \omega) \widehat{G}(j \omega)\right\} \geq \delta_{2} \quad \forall \omega \in \mathbb{R}$

for some $\delta_{2}>0$.

Remark 3.2. If a multiplier $M_{b}$ is a phase-substitute of $M_{a}$, then loosely speaking $M_{a}$ can be substituted by $M_{b}$ in a stability result.

The notion phase-containment is the extension of phasesubstitution for the full class.

Definition 3.3. Let $\mathcal{M}_{A}$ and $\mathcal{M}_{B}$ be two classes of multipliers. The class $\mathcal{M}_{A}$ is phase-contained within the class $\mathcal{M}_{B}$ if given a multiplier $M_{a} \in \mathcal{M}_{A}$, then there exists $M_{b} \in \mathcal{M}_{B}$ such that it is a phasesubstitute of $M_{a}$.

Definitions 3.1 and 3.4 lead naturally to the following definition of equivalence:

Definition 3.4. Two classes of multipliers, $\mathcal{M}_{A}$ and $\mathcal{M}_{B}$, are phaseequivalent if $\mathcal{M}_{A}$ is phase-contained within $\mathcal{M}_{B}$ and $\mathcal{M}_{B}$ is phasecontained within $\mathcal{M}_{A}$.

In the following sections, we will show relationships between different classes of multipliers and the Zames-Falb multipliers:

- The class of Popov multipliers is phase-contained within the class of first order Zames-Falb multipliers, as suggested by Kulkarni and Safonov (2002b). In Section 4, we confirm the relation with mathematical rigour. In particular we show the class of Popov multipliers with positive constant is phase-contained within the class of causal first order Zames-Falb multipliers while the class of Popov multipliers with negative constant is phase-contained within the class of anti-causal first order Zames-Falb multipliers.

- In Section 5 we show the class of SISO multipliers proposed by Park (2002) is phase-equivalent to the class of first order Zames-Falb multipliers.

- In Section 6 we show the classes of multipliers generated by the extensions of the Zames-Falb multipliers given in Altshuller (2011); Jönsson (1997); and Turner and Kerr (2012) are all phase-contained within the class of Zames-Falb multipliers.

\section{Popov multipliers}

Popov multipliers were the first multipliers proposed in the literature (Popov, 1961). Moreover, the cited paper gives the first general solution to the Lur'e problem when the nonlinearity is sector-bounded and time invariant. However, the use of this class of multipliers carries the restriction that the LTI system must be strictly proper and the derivative of the input (depicted $f$ in Fig. 1) must belong to $\mathscr{L}_{2}$ (see Section 6.6.2 in Vidyasagar (1993)).

Definition 4.1. The class of Popov multipliers is given by

$M(s)=1+q s, \quad$ where $q \in \mathbb{R}$.

Remark 4.2. An alternative definition is given by Brockett and Willems (1965):

$M(s)=(1+q s)^{ \pm 1}, \quad$ where $q>0$.

This gives a phase-equivalent class of multiplier.

Since they are not biproper, these are not Zames-Falb multipliers. They have been identified as a limiting case of the Zames-Falb multiplier in Kulkarni and Safonov (2002b) as follows:

$1+q s=\lim _{\epsilon \rightarrow 0^{+}} \frac{1+q s}{1+\epsilon S}$,

$\frac{1}{1+q S}=\lim _{\epsilon \rightarrow 0^{+}} \frac{1+\epsilon S}{1+q S}$.

A careful analysis of both limits shows that the transfer function on the right in (18) is a Zames-Falb multiplier when $\epsilon$ is sufficiently small, but that the transfer function on the right in (19) is not a Zames-Falb multiplier for small $\epsilon$. Moreover the equivalence is not well-defined: at high frequency the Popov multiplier in (18) is unbounded.

Let us first characterize the class of first order Zames-Falb multipliers.

Corollary 4.3. Let $M(s)$ be a first order transfer function given by

$M(s)=\frac{1+v s}{1+\kappa s}$.

Then, $M \in \mathcal{M}$ if and only if $\nu \kappa>0$ and $\left|1-\frac{v}{\kappa}\right|<\frac{v}{\kappa}$.

Proof. Given $v>0$ and $\kappa>0$, then $M(\infty)=\frac{v}{\kappa}$, and $\tilde{M}(s)$ is given by

$\tilde{M}(s)=\frac{1-\frac{v}{\kappa}}{1+\kappa s}$.

Applying Lemma 2.3 and using the relation

$\left\|\frac{1}{1+\kappa s}\right\|_{1}=1$

gives the result. 
It is clear that the limit in (18) is a Zames-Falb multiplier for all

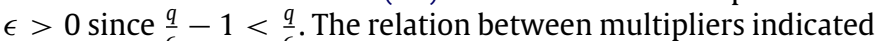
by (18) can be formalized as follows:

Lemma 4.4. The class of Popov multipliers with positive constant $q$ is phase-contained within the class of causal first order Zames-Falb multipliers.

Proof. Assume that there exists some $q>0$ such that

$\operatorname{Re}\{(1+q j \omega) \widehat{G}(j \omega)\} \geq \delta \quad \forall \omega \in \mathbb{R}$,

for some $\delta>0$ and some $\widehat{G} \in \& \mathcal{R}$. Let us take arbitrarily small $\epsilon$ such that $0<\epsilon<q$. Then, the phase-substitute multiplier

$M(s)=\frac{1+q s}{1+\epsilon s}$,

is a Zames-Falb multiplier and it is straightforward that there exists a $\delta_{2}$ such that

$\operatorname{Re}\{M(j \omega) \widehat{G}(j \omega)\}>\delta_{2} \quad \forall \omega \in \mathbb{R}$.

In the case of $v<\kappa$, Corollary 4.3 requires $v<\kappa<2 v$. Thus, the limit in (19) is not a Zames-Falb multiplier as soon as $2 \epsilon \leq q$. Nevertheless, if the class of Popov multipliers is considered by using (17), an appropriate limit with $\epsilon<0$ can be stated:

$1+q s=\lim _{\epsilon \rightarrow 0^{-}} \frac{1+q s}{1+\epsilon s}, \quad q<0$.

Lemma 4.5. The class of Popov multipliers with negative constant $q$ is phase-contained within the class of anti-causal first order Zames-Falb multipliers.

Proof. Similar to Lemma 4.4 but with $\epsilon<0$.

Remark 4.6. We may think of a Popov multiplier as a first order Zames-Falb multiplier but with its pole at infinity.

As a result, a new version of the Popov Theorem can be given as a corollary of Theorem 2.1. We require that the nonlinearity be slope-restricted with $k$ finite. However the LTI system may be biproper and input-output stability is established without further restriction on the derivative of the input:

Corollary 4.7 (Popov Theorem). Let $G \in \mathbf{R H}_{\infty}$ and let $\phi_{k}$ be a sloperestricted $S[0, k]$ nonlinearity. If there exists $q \in \mathbb{R}$ such that

$\operatorname{Re}\{(1+q j \omega)(1+k G(j \omega))\} \geq \delta \quad \forall \omega \in \mathbb{R}$

for some $\delta>0$, then the feedback interconnection (4) is $\mathcal{L}_{2}$ stable.

\section{Park's multipliers}

Park (2002) proposes a class of multipliers which corresponds to a stability condition that can be tested by a convex search. It is easy to show the phase-equivalence between this class of multipliers and the class of first order Zames-Falb multipliers. The multipliers are given by

$M(s)=-s^{2}+a^{2}+b s, \quad a \in \mathbb{R}, b \in \mathbb{R}$.

The quadratic term was introduced by Yakubovich $(1965)^{2}$ where the frequency condition was obtained by using a Lur'e-Postnikov

\footnotetext{
2 Other authors (Altshuller, 2011; Barabanov, 1988) cite a conference paper in 1962 now unavailable.
}

type Lyapunov function. A similar result was independently developed by Dewey and Jury (1966) within an embryonic passivity framework. Barabanov (1988) uses the same class of multipliers to demonstrate the Kalman conjecture for third order systems.

As commented by Park (2002), a proper multiplier with the same phase can be defined as follows:

Definition 5.1. The class of Park's multipliers is given by

$M_{P}(s)=1+\frac{b s}{-s^{2}+a^{2}}$

where $a$ and $b$ are real numbers.

Not all multipliers in this class are Zames-Falb multipliers. However the following results provide the equivalence to the class of first order Zames-Falb multipliers. A prior result is needed in order to show that all first order Zames-Falb multipliers are phaseequivalent to a subset of the first order Zames-Falb multipliers.

Lemma 5.2. The class of first order Zames-Falb multipliers is phaseequivalent to the subset of first order Zames-Falb multiplier given by

$M(s)=\frac{s+a}{s+b}$

such that $a b>0$ and $|a|<|b|$.

Proof. We need to show that the first order Zames-Falb multiplier which are not contained within the subset can be substituted by a element within the subset. Let us consider a first order Zames-Falb multiplier

$M(s)=\frac{s+a}{s+b}$

where $a b>0$, and assume that $|a|>|b|$ and

$\operatorname{Re}\{M(j \omega) \widehat{G}(j \omega)\}>\delta_{1} \quad \forall \omega \in \mathbb{R}$,

for some $\widehat{G} \in \& \mathcal{R}$. Then, its phase-substitute Zames-Falb multiplier is

$M^{\prime}(s)=\frac{s-b}{s-a}$,

where Corollary 4.3 can be applied in order to prove that $M^{\prime} \in \mathcal{M}$ and

$\frac{(j \omega+a)(-j \omega+a)}{(j \omega+b)(-j \omega+b)} \operatorname{Re}\left\{M^{\prime}(j \omega) \widehat{G}(j \omega)\right\}>\delta_{1} \quad \forall \omega \in \mathbb{R}$.

Since there exists $\xi$ such that

$\frac{1}{\xi}>\frac{(j \omega+a)(-j \omega+a)}{(j \omega+b)(-j \omega+b)}>\xi \quad \forall \omega \in \mathbb{R}$

it follows that there exists $\delta_{2}$ such that

$\operatorname{Re}\left\{M^{\prime}(j \omega) \widehat{G}(j \omega)\right\}>\delta_{2} \quad \forall \omega \in \mathbb{R}$.

An equivalence result follows:

Lemma 5.3. The class of Park's multipliers is phase-equivalent to the class of first order Zames-Falb multipliers.

Proof. Let $M_{P}$ be a Park's multiplier such that

$\operatorname{Re}\left\{M_{P}(j \omega) \widehat{G}(j \omega)\right\}>\delta_{1} \quad \forall \omega \in \mathbb{R}$, 
for some $\delta_{1}$ and some $\widehat{G} \in \& \mathcal{R}$ and let $z_{1}$ and $z_{2}$ be the roots of $-s^{2}+b s+a^{2}$. If $b>0$, then

$z_{1}=\frac{b-\sqrt{b^{2}+4 a^{2}}}{2}$

$z_{2}=\frac{b+\sqrt{b^{2}+4 a^{2}}}{2}$,

and if $b<0$

$z_{1}=\frac{b+\sqrt{b^{2}+4 a^{2}}}{2}$

$z_{2}=\frac{b-\sqrt{b^{2}+4 a^{2}}}{2}$.

Note that $\left|z_{1}\right|<\left|z_{2}\right|$. Then, (37) is equivalent to the condition

$\operatorname{Re}\left\{-\frac{\left(j \omega-z_{1}\right)\left(j \omega-z_{2}\right)}{(a+j \omega)(a-j \omega)} \widehat{G}(j \omega)\right\}>\delta_{1} \quad \forall \omega \in \mathbb{R}$

or equivalently

$\operatorname{Re}\left\{-\frac{\left(j \omega-z_{1}\right)\left(j \omega-z_{2}\right)}{(a+j \omega)(a-j \omega)} \frac{\left(-j \omega-z_{2}\right)}{\left(-j \omega-z_{2}\right)} \widehat{G}(j \omega)\right\}>\delta_{1}$

for all $\omega \in \mathbb{R}$. This can be rewritten as

$\operatorname{Re}\left\{\frac{j \omega-z_{1}}{j \omega+z_{2}} \frac{\left(j \omega-z_{2}\right)\left(-j \omega-z_{2}\right)}{(a+j \omega)(a-j \omega)} \widehat{G}(j \omega)\right\}>\delta_{1}$

for all $\omega \in \mathbb{R}$. But there exists $\xi$ such that

$\frac{1}{\xi}>\frac{\left(j \omega-z_{2}\right)\left(-j \omega-z_{2}\right)}{(a+j \omega)(a-j \omega)}>\xi>0$.

Hence, it follows that (37) is satisfied if and only if there exists some $\delta_{2}>0$ such that

$\operatorname{Re}\left\{\frac{j \omega-z_{1}}{j \omega+z_{2}} \widehat{G}(j \omega)\right\}>\delta_{2} \quad \forall \omega \in \mathbb{R}$.

Since $z_{1} z_{2}<0$ and $\left|z_{1}\right|<\left|z_{2}\right|$, the application of Lemma 2.3 leads to

$M(s)=\frac{s-z_{1}}{s+z_{2}} \in \mathcal{M}$.

Finally, we need to prove the necessity. The result is straightforwardly obtained by using Lemma 5.2.

Remark 5.4. A more detailed classification can be extracted from (39) and (46). Given a Park's multiplier with $b>0$, its phasesubstitute Zames-Falb multiplier is causal, whereas if $b<0$, its phase-substitute Zames-Falb multiplier is anticausal.

The stability result proposed in Park (2002) now follows as a corollary of Theorem 2.1 and can be stated in terms of $\mathcal{L}_{2}$-stability without a requirement that the LTI plant be strictly proper.

Corollary 5.5. Let $G \in \mathbf{R H}_{\infty}$ and let $\phi_{k}$ be a slope-restricted $S[0, k]$ nonlinearity. If there exists a multiplier $M_{p}$ of the form (29) such that

$\operatorname{Re}\left\{M_{p}(j \omega)(1+k G(j \omega))\right\} \geq \delta \quad \forall \omega \in \mathbb{R}$

for some $\delta>0$, then the feedback interconnection (4) is $\mathcal{L}_{2}$ stable.

In summary, the result given in Park (2002) can be understood as an LMI search over the whole class of first order rational Zames-Falb multipliers.

\section{Extensions of Zames-Falb multipliers}

Three different extensions of the class of Zames-Falb multipliers have been proposed in the literature: adding a "Popov term", i.e. qs, (Jönsson, 1997), adding a Popov multiplier (Turner \& Kerr, 2012) and adding a "Yakubovich term" i.e. $-\kappa^{2} s^{2}$ (Altshuller, 2011). For SISO systems, we show that all three extensions are phasecontained within the original class, and hence any additional conditions associated with the extra terms are not needed.

\subsection{Extension adding the Popov term}

The extended class is defined as follows:

Definition 6.1. The class of Popov-extended Zames-Falb multipliers is given by

$M_{\mathrm{PZF}}(s)=q s+M(s)$

where $q \in \mathbb{R}$ and where $M(s)$ belongs to the class of Zames-Falb multipliers.

Lemma 6.2. The class of Popov-extended Zames-Falb multipliers is phase-contained within the class of Zames-Falb multiplier.

Proof. Given a multiplier $M \in \mathcal{M}$ then $M(s)=1+H(s)$ for some strictly proper transfer function $H(s)$ with $\|H(s)\|_{1}<1$. Then there exists $\rho>0$ such that $\|H(s)\|_{1}<1-\rho$. Thus,

$M(s)=\rho+((1-\rho)+H(s))=\rho+M^{\prime}(s)$

where $M^{\prime}(s)$ is a Zames-Falb multiplier. Hence (49) can be rewritten as follows

$M_{\mathrm{PZF}}(s)=\rho\left(1+\frac{q}{\rho} s\right)+(M(s)-\rho)$.

The argument is similar to the proof of Lemma 4.4. Let $\widehat{G} \in \& \mathcal{R}$. There is some $\delta_{1}>0$ such that $M_{\text {PZF }}$ satisfies

$\operatorname{Re}\left\{M_{\text {PZF }}(j \omega) \widehat{G}(j \omega)\right\}>\delta_{1} \quad \forall \omega \in \mathbb{R}$.

For $\epsilon$ sufficiently small chosen such that $\epsilon q>0$, the multiplier given by

$M_{2}(s)=\rho\left(\frac{1+\frac{q}{\rho} s}{1+\epsilon s}\right)+M_{1}^{\prime}(s)$,

is a Zames-Falb multiplier (since the sum of two Zames-Falb multipliers is also a Zames-Falb multiplier) and satisfies

$\operatorname{Re}\left\{M_{2}(j \omega) \widehat{G}(j \omega)\right\}>\delta_{2} \quad \forall \omega \in \mathbb{R}$,

with $\delta_{2}>0$.

Remark 6.3. Jönsson (1997) suggests that such an extension can give better results. The apparent contradiction here is associated with our restriction that $\hat{G}$ should be biproper, whereas the example of Jönsson (1997) is strictly proper. See Carrasco, Heath, and Lanzon (2013) for further discussion.

We can state the result given by Jönsson (1997) as a corollary of Theorem 2.1, avoiding further conditions normally imposed by the use of a Popov multiplier.

Corollary 6.4. Let $G \in \mathbf{R H}_{\infty}$ and let $\phi_{k}$ be a slope-restricted $S[0, k]$ nonlinearity. Assume that there exists a Popov-extended Zames-Falb multiplier $M_{\mathrm{PZF}}$ such that

$\operatorname{Re}\left\{M_{\mathrm{PZF}}(j \omega)(1+k G(j \omega))\right\} \geq \delta \quad \forall \omega \in \mathbb{R}$

for some $\delta>0$. Then the feedback interconnection (4) is $\mathcal{L}_{2}$ stable. 


\subsection{Extension adding a Popov multiplier}

A convex search over a class of causal Zames-Falb multipliers is proposed by Turner et al. (2009). A further proposal to add a Popov multiplier gives improved results (Turner \& Kerr, 2012). See also Carrasco, Heath, Li et al. (2012) and Turner, Kerr, and Postlethwaite (2012) for discussion.

Definition 6.5. The class of Popov plus Zames-Falb multipliers is given by

$M_{\mathrm{P}+\mathrm{ZF}}(s)=\vartheta(1+q s)+M(s)$

where $q \in \mathbb{R}$, where $\vartheta>0$ and where $M(s)$ belongs to the class of Zames-Falb multipliers.

Lemma 6.6. The class of Popov plus Zames-Falb multipliers is phasecontained within the class of Zames-Falb multipliers.

Proof. Let us take a Popov plus Zames-Falb multiplier given by

$M_{\mathrm{P}+\mathrm{ZF}}(s)=\vartheta+\eta s+M(s) \quad \vartheta>0 \eta \in \mathbb{R}$,

where $M(s)$ is a Zames-Falb multiplier, i.e.

$M_{\mathrm{P}+\mathrm{ZF}}(s)=\vartheta+\eta s+1+H(s)$,

where $\|H\|_{1}<1$. Then $M_{\mathrm{P}+\mathrm{ZF}}$ can be rewritten as a multiplier consider in the previous section as follows

$M_{\mathrm{P}+\mathrm{ZF}}(s)=(\vartheta+1)\left(\frac{\eta}{\vartheta+1} s+1+\frac{H(s)}{\vartheta+1}\right)$,

where the proportional constant can trivially be ignored. Since $\vartheta>0$, then

$\left\|\frac{H(s)}{\vartheta+1}\right\|_{1}<1$

yielding

$M_{\mathrm{P}+\mathrm{ZF}}(s)=(\vartheta+1) M_{\mathrm{PZF}}(s)$.

Therefore, the result is obtained by applying Lemma 6.2.

Remark 6.7. Turner and Kerr (2012) claim that a search over the class of Popov plus Zames-Falb multipliers can have significant improvement over the search proposed by Turner et al. (2009). Lemma 6.6 does not contradict the result presented by Turner and Kerr (2012). The original search of Turner et al. (2009) is carried out within the class of causal Zames-Falb multipliers, whereas the search over the class of Popov plus Zames-Falb multipliers can result in a noncausal Zames-Falb multiplier if $q<0$, as shown in Lemma 4.5.

Once again, the following corollary of Theorem 2.1 relaxes the conditions for applying Proposition 1 of Turner and Kerr (2012) and provides an $\mathscr{L}_{2}$-stability result.

Corollary 6.8. Let $G \in \mathbf{R H}_{\infty}$ and let $\phi_{k}$ be a slope-restricted $S[0, k]$ nonlinearity. Assume that there exists a Popov plus Zames-Falb multiplier $M_{\mathrm{P}+\mathrm{ZF}}$ such that

$\operatorname{Re}\left\{M_{\mathrm{P}+\mathrm{ZF}}(j \omega)(1+k G(j \omega))\right\} \geq \delta \quad \forall \omega \in \mathbb{R}$

for some $\delta>0$. Then the feedback interconnection (4) is $\mathcal{L}_{2}$-stable.

Remark 6.9. Although not required in Corollary 6.8, a condition that $G$ be strictly proper is required for the derivation of the LMI given by Eq. (3) of Turner and Kerr (2012) since the state space representation of $s G(s)$ is used.

\subsection{Extension with "Yakubovich term"}

Using Theorem 3 in Altshuller (2011) for SISO systems, the class of Zames-Falb multipliers can be extended as follows:

Definition 6.10. The class of Yakubovich-Zames-Falb multipliers is given by

$M_{\mathrm{YZF}}(s)=-\kappa^{2} s^{2}+M(s), \quad \kappa \in \mathbb{R}$,

where $\kappa \in \mathbb{R}$ and $M(s)$ is a Zames-Falb multiplier.

Lemma 6.11. The class of Yakubovich-Zames-Falb multiplier is phase-contained with the class of Zames-Falb multipliers.

Proof. We have already observed that $M(j \omega)$ in (28) has the same phase as $M_{P}(j \omega)$ in (29); see also Corollary 4 and Corollary 5 in Dewey and Jury (1966). Similarly, since $\left(1+\kappa^{2} \omega^{2}\right)>1$ for all $\omega \in \mathbb{R}$, it is straightforward to show that the class defined by (63) is phase-equivalent to the class defined by

$M_{\mathrm{YZF}}(s)=1+\frac{H(s)}{1-\kappa^{2} s^{2}}$,

where $H(s)$ is a strictly proper transfer function such that $\|H\|_{1}<$ 1. Since $\left\|H_{1} H_{2}\right\|_{1} \leq\left\|H_{1}\right\|_{1}\left\|H_{2}\right\|_{1}$ (Lemma 12 in Vidyasagar (1993, p. 295)), it follows that

$\left\|\frac{H}{1-\kappa^{2} s^{2}}\right\|_{1} \leq\|H\|_{1}\left\|\frac{1}{1-\kappa s}\right\|_{1}\left\|\frac{1}{1+\kappa s}\right\|_{1}<1$.

Hence, the multiplier $M_{\mathrm{YZF}}(s)$ is a Zames-Falb multiplier.

Finally, the following corollary of Theorem 2.1 is less restrictive that the SISO version of the result given by Altshuller (2011).

Corollary 6.12. Let $G \in \mathbf{R H}_{\infty}$ and let $\phi_{k}$ be a slope-restricted $S[0, k]$ nonlinearity. Assume that there exists a Yakubovich Zames-Falb multiplier $M_{\mathrm{YZF}}$ such that

$\operatorname{Re}\left\{M_{\mathrm{YZF}}(j \omega)(1+k G(j \omega))\right\} \geq \delta \quad \forall \omega \in \mathbb{R}$

for some $\delta>0$. Then the feedback interconnection (4) is $\mathcal{L}_{2-}$ stable.

\section{Comments on the classes of multipliers}

Our results prompt the natural questions: are multipliers such as the Popov multiplier and Park's multiplier redundant when the nonlinearity is slope-restricted and are the extensions useless? The answers remain an emphatic no.

Consider for example the class of Park's multipliers. We have shown (Lemma 5.3) that it is phase-equivalent to the class of first order Zames-Falb multipliers (note in passing that this gives an independent proof of Theorem 2 in Park (2002)). We have also observed that Park's multipliers have been discovered many times in the literature (Barabanov, 1988; Dewey \& Jury, 1966; Yakubovich, 1965). Nevertheless the main contribution of Park (2002) remains: a parametrization of the first order Zames-Falb multipliers that is amenable to convex search. It is for this reason that the stability test of Park (2002) has become a benchmark (e.g. Turner et al., 2009) despite appearing in the literature more than 30 years after the original work of Zames and Falb (1968). We emphasize that it is the parametrization of the class of multiplier rather than the class itself that is novel.

In Turner and Kerr (2012); and Turner et al. (2012), a search over the class of Popov-Zames-Falb multipliers shows promising results. As demonstrated in Section 6, there exists an equivalent Zames-Falb multiplier with the same properties as any such 
Popov-Zames-Falb multiplier, but a convex search over the equivalent Zames-Falb multipliers is not yet known. A similar search over anticausal Zames-Falb multipliers has been proposed by Carrasco, Maya-Gonzalez, Lanzon, and Heath (2012) and its extension to Popov-Zames-Falb multipliers is also possible. They appear to be the most competitive searches over Zames-Falb multipliers. Therefore, extensions are very useful to provide better parametrizations of the class of Zames-Falb multipliers.

\section{Conclusion}

In order to analyse the relationships between different classes of multipliers, notions of phase-containment and phaseequivalence have been defined. Most of the classes of multipliers defined in the literature for slope-restricted nonlinearities, such as Popov multipliers, Park's multipliers, and the extensions of Zames-Falb multipliers, are discussed in this paper. Lemmas 4.4, $4.5,5.3,6.2,6.6$ and 6.11 show that all these classes of multipliers are phase-contained within the class of Zames-Falb multipliers. This provides new $\mathcal{L}_{2}$-stability results as corollaries of Theorem 2.1. The only conditions required are those which are given for the Zames-Falb multipliers (Zames \& Falb, 1968). Corollaries 4.7, 5.5, 6.4 and 6.12 are all believed to be novel.

No convex search over the whole class of Zames-Falb multipliers has yet been found. This can be largely ascribed to difficulties associated with the bound on the $\mathcal{L}_{1}$-norm in the original definition. Hence from a practical point of view, the classes of multipliers discussed in this paper remain useful. The analysis of this paper indicates that any improvement from their use should be interpreted as arising from a convenient parametrization within the class of Zames-Falb multipliers.

\section{Acknowledgements}

We would like to thank the anonymous reviewers and associate editor for their many helpful comments and suggestions.

\section{References}

Altshuller, D. (2011). Delay-integral-quadratic constraints and stability multipliers for systems with MIMO nonlinearities. IEEE Transactions on Automatic Control 56(4), 738-747.

Barabanov, N. E. (1988). On the Kalman problem. Siberian Mathematical Journal, 29, 333-341.

Brockett, R., \& Willems, J. (1965). Frequency domain stability criteria-part I. IEEE Transactions on Automatic Control, 10(3), 255-261.

Carrasco, J., Heath, W. P., \& Lanzon, A. (2012). Factorization of multipliers in passivity and IQC analysis. Automatica, 48(5), 909-916.

Carrasco, J., Heath, W.P., \& Lanzon, A. (2013). On multipliers for bounded and monotone nonlinearities, to be presented at the 12th biannual European Control Conference, 2013.

Carrasco, J., Heath, W. P., Li, G., \& Lanzon, A. (2012). Comments on on the existence of stable, causal multipliers for systems with slope-restricted nonlinearities. IEEE Transactions on Automatic Control, 57(9), 2422-2428.

Carrasco, J., Maya-Gonzalez, M., Lanzon, A., \& Heath, W.P. (2012). LMI search for rational anticausal Zames-Falb multipliers. In 51th control and decision conference (pp. 7770-7775).

Chang, M., Mancera, R., \& Safonov, M. (2011). Computation of Zames-Falb multipliers revisited. IEEE Transactions on Automatic Control, 57(4), 1024-1029.

Chen, X., \& Wen, J. (1995). Robustness analysis of LTI systems with structured incrementally sector bounded nonlinearities. In Proceedings of the American control conference, 1995, Vol. 5 (pp. 3883-3887).

Cho, Y.-S., \& Narendra, K. (1968). An off-axis circle criterion for stability of feedback systems with a monotonic nonlinearity. IEEE Transactions on Automatic Control, 13(4), 413-416.

D’Amato, F. J., Rotea, M. A., Megretski, A. V., \& Jönsson, U. T. (2001). New results for analysis of systems with repeated nonlinearities. Automatica, 37(5), 739-747.

Desoer, C. A., \& Vidyasagar, M. (1975). Feedback systems: input-output properties. Orlando, FL, USA: Academic Press, Inc.

Dewey, A., \& Jury, E. (1966). A stability inequality for a class of nonlinear feedback systems. IEEE Transactions on Automatic Control, 11(1), 54-62.

Falb, P., \& Zames, G. (1968). Multipliers with real poles and zeros: an application of a theorem on stability conditions. IEEE Transactions on Automatic Control, 13(1), $125-126$.
Gapski, P., \& Geromel, J. (1994). A convex approach to the absolute stability problem. IEEE Transactions on Automatic Control, 39(9), 1929-1932.

Gruber, M. (1969). Path integrals and Lyapunov functionals. IEEE Transactions on Automatic Control, 14(5), 465-475.

Heath, W. P., \& Wills, A. G. (2007). Zames-Falb multipliers for quadratic programming. IEEE Transactions on Automatic Control, 52(10), 1948-1951.

Jönsson, U. T. (1997). Stability analysis with Popov multipliers and integral quadratic constraints. Systems \& Control Letters, 31(2), 85-92.

Kerr, M. L., Turner, M. C., Villota, E., Jayasuriya, S., \& Postlethwaite, I. (2011). A robust anti-windup design procedure for SISO systems. International Journal of Control, 84(2), 351-369.

Khalil, H. K. (2002). Nonlinear systems. Prentice Hall.

Kothare, M. V., \& Morari, M. (1999). Multiplier theory for stability analysis of antiwindup control systems. Automatica, 35(5), 917-928.

Kulkarni, V., \& Safonov, M. (2002a). All multipliers for repeated monotone nonlinearities. IEEE Transactions on Automatic Control, 47(7), 1209-1212.

Kulkarni, V., \& Safonov, M. (2002b). Incremental positivity nonpreservation by stability multipliers. IEEE Transactions on Automatic Control, 47(1), 173-177.

Megretski, A., \& Rantzer, A. (1997). System analysis via integral quadratic constraints. IEEE Transactions on Automatic Control, 42(6), 819-830.

Moreno, J. C., Baños, A., \& Berenguel, M. (2010). A QFT framework for antiwindup control systems design. Journal of Dynamic Systems, Measurement, and Control, 132(2), 021012.

Mulder, E., Kothare, M., \& Morari, M. (2001). Multivariable anti-windup controller synthesis using linear matrix inequalities. Automatica, 37(9), 1407-1416.

Park, P. (2002). Stability criteria of sector- and slope-restricted Lur'e systems. IEEE Transactions on Automatic Control, 47(2), 308-313.

Popov, V. (1961). Absolute stability of nonlinear systems of automatic control. Automation and Remote Control, 22(8), 857-875.

Safonov, M., \& Wyetzner, G. (1987). Computer-aided stability analysis renders Popov criterion obsolete. IEEE Transactions on Automatic Control, 32(12), 1128-1131.

Turner, M. C., \& Kerr, M. L. (2012). $\mathscr{L}_{2}$ gain bounds for systems with sector bounded and slope-restricted nonlinearities. International Journal of Robust and Nonlinear Control, 22(13), 1505-1521.

Turner, M. C., Kerr, M. L., \& Postlethwaite, I. (2009). On the existence of stable, causal multipliers for systems with slope-restricted nonlinearities. IEEE Transactions on Automatic Control, 54(11), 2697-2702.

Turner, M. C., Kerr, M. L., \& Postlethwaite, I. (2012). Authors reply to comments on 'on the existence of stable, causal multipliers for systems with slope-restricted nonlinearities'. IEEE Transactions on Automatic Control, 57(9), 2428-2431.

Veenman, J., \& Scherer, C.W. (2011). IQC-synthesis with general dynamic multipliers. In 18th IFAC world congress.

Vidyasagar, M. (1993). Nonlinear systems analysis. London: Prentice-Hall International Editions.

Willems, J. C. (1998). Path integrals and stability. In J. Baillieul, \& J. Willems (Eds.), Mathematical control theory (pp. 1-37). Springer Verlag.

Yakubovich, V. (1965). Frequency conditions for the absolute stability and dissipativity of control systems with a single differentiable nonlinearity. Soviet Mathematics Doklady, 6, 98-101.

Zames, G. (1966). On the input-output stability of time-varying nonlinear feedback systems-part II: conditions involving circles in the frequency plane and sector nonlinearities. IEEE Transactions on Automatic Control, 11(3), 465-476.

Zames, G., \& Falb, P. L. (1968). Stability conditions for systems with monotone and slope-restricted nonlinearities. SIAM Journal on Control, 6(1), 89-108.

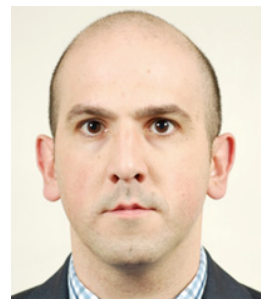

Joaquin Carrasco is a Lecturer at the Control Systems Centre, School of Electrical and Electronic Engineering, University of Manchester, UK He was born in Abarán, Spain, in 1978. He received the B.Sc. degree in physics and the Ph.D. degree in control engineering from the University of Murcia, Murcia, Spain, in 2004 and 2009, respectively. From 2009 to 2010, he was with the Institute of Measurement and Automatic Control, Leibniz Universität Hannover, Hannover, Germany. From 2010 to 2011, he was a research associate at the Control Systems Centre, School of Electrical and Electronic Engineering, University of Manchester, Manchester, UK He has been a Visiting Researcher at the University of Groningen, Groningen, The Netherlands, and the University of Massachusetts, Amherst. His research interests include multiplier theory and passive systems.

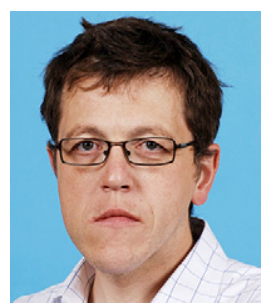

William P. Heath is a Reader at the School of Electrical and Electronic Engineering, University of Manchester, UK and is Head of the Control Systems Centre at the University. His interests include constrained control and system identification. He has a B.A. and M.A. in Mathematics from Cambridge University, and an M.Sc. and a Ph.D. from UMIST. Before his appointment at Manchester he spent several years in both industry at Lucas Automotive and academia at the University of Newcastle, Australia. 


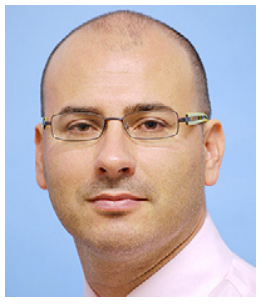

Alexander Lanzon is a Reader in Control Engineering at the School of Electrical and Electronic Engineering and the Autonomous Systems Theme Leader at the Aerospace Research Institute, both at the University of Manchester. He was born in Malta. He received the B.Eng. (Hons). degree in Electrical and Electronic Engineering with highest-level First Class Honours from the University of Malta (Malta) in 1995, and his M.Phil. degree in Robotics and his Ph.D. degree in Control Engineering from the University of Cambridge (UK) in 1997 and 2000 respectively.

Before joining the School of Electrical and Electronic Engineering at the University of Manchester in December 2006, Alexander held academic positions at the School of Aerospace Engineering, Georgia Institute of Technology, USA, and the Australian National University, Australia. Dr. Lanzon also received earlier research training at Bauman Moscow State Technical University, Russia and industrial training at ST-Microelectronics Ltd., Malta, and Yaskawa Denki Tokyo Ltd., Japan. In Australia, Alexander held also the position of senior research scientist at National ICT Australia Ltd.

Dr. Lanzon is an Associate Editor of the IEEE Transactions on Automatic Control, a Subject Editor of the International Journal of Robust and Nonlinear Control, Fellow of the IET, and a Senior Member of both the IEEE and the AIAA. He has authored and co-authored over 120 articles and has managed or co-managed over GBP $6 M$ in research grant contracts. His current research interests include the fundamental theory of robust feedback control systems and aerospace control applications, particularly unmanned autonomous aerial/underwater vehicles. 